\title{
INTOXICACIÓN POR ACETAMINOFÉN
}

\author{
Liliana C. Mancipe MD. ${ }^{1,}$ Diana C. Fernández A. MD. ${ }^{2} \gamma$ Daniel G. Fernández A. MD. ${ }^{3}$ \\ ${ }^{1}$ Médica Fisiatra Hospital Militar Central - Clínica Policía Nacional, ${ }^{2}$ Cirujana Universidad Pedagógica y \\ Tecnológica de Colombia, Médica Servicio Social Obligatorio Clínica de la Policía Nacional - Tunja, ${ }^{3}$ Internista y \\ Reumatólogo Hospital Universitario San Ignacio. Docente Facultad de Medicina Pontificia Universidad Javeriana
}

\section{Resumen}

El acetaminofén es uno de los analgésicos de más amplio uso en el mundo. Su buen perfil de seguridad, eficacia y el hecho de ser de un medicamento de venta libre, hacen que la formulación y la utilización de este fármaco sea muy común, tanto en la población adulta como en la pediátrica. Los cuadros de intoxicación por acetaminofén son causa frecuente de consulta en los servicios de urgencias; por tal razón, los médicos de estas áreas deben tener un claro conocimiento sobre la fisiopatología, cuadro clínico, diagnóstico y tratamiento. En el presente artículo se hace una revisión sobre el diagnóstico y el tratamiento de los pacientes intoxicados con este medicamento.

Palabras clave: acetaminofén, toxicidad, hígado, fallo hepático

\section{ACETAMINOPHEN POISONING}

\begin{abstract}
Acetaminophen is one of the most widely used analgesics in the world. Its good safety profile, efficacy and the fact that it is on the shelf medicine, makes the formulation of this drug very common in both adult and children. Acetaminophen poisoning is a frequent motive of consultation in the emergency department, so that doctors in these areas must have a clear understanding of the pathophysiology, clinical presentation, diagnosis and treatment of this type of poisoning. In this article we make an update on the diagnosis and treatment of acetaminophen-poisoned patients.
\end{abstract}

Key words: acetaminophen, toxicity, liver, liver failure

\section{INTOXICAÇÃO PELO ACETAMINOFEN}

\section{Resumo}

O acetaminofeno é um dos analgésicos mais utilizados no mundo. Seu bom perfil de segurança, a eficácia eo fato de que um medicamento de venda livre, fazem que a formulação e utilização desta droga seja muito comum tanto na população adulta como a pediátrica. As manifestações de intoxicação por acetaminofen são causas freqüentes de consulta na unidade de emergência; Por esta razão, os médicos nessas áreas devem ter uma compreensão clara da fisiopatologia, aspectos clínicos, diagnóstico e tratamento. Neste artigo se faz uma revisão do diagnóstico e tratamento de pacientes intoxicados com essa droga.

Palabras-chave: acetaminofen, toxicidade, fígado, falencia hepática

* Correspondencia: Daniel Fernández danielfernandezmd@gmail.com. Dirección postal: Tr. 3 № 49-00. Facultad de Medicina, Universidad Militar Nueva Granada. Bogotá, Colombia.

Recibido: Febrero 24 de 2010 Aceptado: Diciembre 1 de 2010 


\section{Introducción}

El acetaminofén, introducido en la medicina en 1893 por Von Mering, es uno de los analgésicos y antipiréticos de mayor uso clinico y domiciliario debido a su libre comercialización, su alta efectividad y su bajo costo, lo cual hace más fácil que sea uno de los principales agentes causantes de sobredosis accidentales e intencionales que conllevan a consultar a los servicios de Urgencias.

Este medicamento fue aprobado por la FDA (Food and Drug Administration) en 1960, como tableta de liberación inmediata de $325 \mathrm{mg}$. Las cápsulas y tabletas de $500 \mathrm{mg}$ fueron aprobadas en 1973 y 1975 respectivamente. Basados en la literatura disponible, la FDA en Estados Unidos ha determinado que el acetaminofén es seguro y efectivo a una dosis máxima diaria de 4 gramos en 24 horas (1).

\section{Epidemiología}

El acetaminofén es el agente farmacéutico más investigado en los centros de intoxicación de Estados Unidos (2) y es la sustancia más comúnmente utilizada para suicidios en el Reino Unido (3). En el año 2003, en Estados Unidos las estadísticas reportadas por la Asociación Americana de Centros de Control de Intoxicaciones, presentan una cifra de aproximadamente 127.000 intoxicaciones por acetaminofén o productos que lo contienen, de las cuales 38.989 ocurrieron en niños menores de seis años (4). En este mismo año se reportaron 214 muertes por sobredosis de medicamentos analgésicos, en 62 de estos casos, el acetaminofén fue el único agente involucrado.

Estas estadísticas se incrementaron en el 2005, año en el que se reportaron 165.000 nuevos casos, de los cuales un $40 \%$ se presentó por ingesta de acetaminofén solo y un $60 \%$ por el consumo de mezclas de acetaminofén con otros fármacos, especialemente opioides (5). La toxicidad por acetaminofén representa la mayor causa de falla hepática fulminante (6) y se observó un incremento alarmante del $21 \%$ al $51 \%$ de este daño hepático entre los años 1988 y 2003; más de la mitad de estos casos se presentaron por sobredosis accidentales (7).

El daño renal es comúnmente observado en los casos de hepatotoxicidad inducida por acetaminofén. Varios estudios han mostrado elevación de la creatinina con valores > 2,0 mg/dl en el $57 \%$ de los pacientes con encefalopatía y coagulopatía secundaria y con falla hepática fulminante secundaria a acetaminofén (7). En Colombia no hay datos epidemiológicos disponibles de este tipo de intoxicaciones; sin embargo, la consulta en los servicios de urgencias de pacientes con complicaciones por sobredosis de acetaminofén es frecuente.

\section{Farmacología y Fisiopatología}

El acetaminofén, un fármaco derivado de la fenacetina, tiene una excelente biodisponibilidad; el pico de concentración plasmática se presenta después de 30 a 60 minutos de su administración por vía oral y su vida media es de dos horas. Presenta metabolismo hepático, en el que se conjuga con ácido glucurónico $(60 \%)$, ácido sulfúrico (35\%) o cisteína (3\%); una pequeña proporción del fármaco presenta $\mathrm{N}$ hidroxilación por el citocromo P450 hasta formar Nacetil-p-benzoquinoneimina (NAPQI), un metabolito potencialmente tóxico, que en condiciones normales es detoxificado mediante conjugación con el glutatión y grupos sulfidrilos (8).

Se ha establecido que la base de la toxicidad por acetaminofén es el consumo de altas dosis, que hace que el citocromo $\mathrm{P} 450$ produzca cantidades de NAPQI capaces de agotar las reservas hepáticas de glutatión. Esto permite que este metabolito tóxico se una a macromoléculas y genere radicales libres, alterando la homeostasis e iniciando la apoptosis de las células del tejido hepático como las del tejido renal, produciéndose necrosis tisular y disfunción orgánica (9-10). El desarrollo de la necrosis hepática se produce en doce horas (11-12).

Además de los efectos a nivel hepático, se presentan otras complicaciones que no se encuentran ampliamente descritas en la literatura, como es el compromiso renal, que puede llevar a falla renal a $1-2 \%$ de los pacientes con sobredosis de acetaminofén (13). La fisiopatología de la toxicidad renal ha sido atribuida a isoenzimas del citocromo $\mathrm{P} 450$ presentes principalmente en la corteza renal; también se han descrito otros mecanismos, como el papel de la prostaglandin endoperóxido sintetasa (PGES), que convierte el acetaminofén en metabolitos tóxicos, especialmente el NAPQI a nivel de la médula renal, y el de las enzimas $\mathrm{N}$-deacetilasas, pero no está bien descrito cual es su mecanismo. Aunque el glutatión se ha considerado como un importante elemento de detoxificación de 
los metabolitos del acetaminofén, paradójicamente su conjugación puede estar implicada en la formación de compuestos nefrotóxicos (14).

\section{Factores de Riesgo}

Dentro de los factores de riesgo que aumentan la toxicidad del medicamento se encuentran el consumo de sustancias inductoras del citocromo P450 (carbamazapina, fenitoína, zidovudina, rifampicina, fenobarbital etc), el consumo de fármacos que compiten por la conjugación con el glutatión (morfina, estrógenos, salicilatos, prednisona etc), los mecanismos que causen depleción de glutatión (malnutrición, HIV, fibrosis quística) y el consumo crónico de etanol $(21 \mathrm{U} /$ semana en hombres y $14 \mathrm{U} /$ semana en mujeres). El consumo de etanol altera el metabolismo del acetaminofén por dos mecanismos: uno es agotar las reservas de glutation y el otro, induce al citocromo P450 y entonces se aumentan la proporción de NAPQI.

\section{Enfoque diagnóstico}

El reconocimiento temprano de la toxicidad por acetaminofén es esencial para disminuir la morbimortalidad de estos pacientes; sin embargo, este reconocimiento puede ser difícil debido a que los hallazgos tempranos en este tipo de intoxicación no son específicos (15). Por tanto, la primera herramienta diagnóstica en urgencias debe ser elaborar una buena encuesta, para que de la información obtenida se pueda sospechar la causa; también es importante conocer los antecedentes del paciente.

\section{Cuadro Clínico}

El cuadro clínico clásico de una intoxicación aguda por acetaminofén se presenta en cuatro etapas que se resumen en la tabla 1.

En los países occidentales, la principal causa de la falla hepática aguda, definida por el inicio de coagulopatía (INR > 1,5) y cambios en el estado mental, se debe a sobredosis por acetaminofén. La mayor parte de estos pacientes desarrollan además complicaciones infecciosas, cardiopulmonares o renales que pueden progresar a falla multiorgánica. El $70 \%$ de los pacientes con transplante hepático sobreviven por un año; sin embargo, menos del $10 \%$ de los pacientes con falla hepática aguda son candidatos para transplante y de estos, más del $20 \%$ fallecen esperando el procedimiento quirúrgico (18).
Tabla 1. Cuadro Clínico en Intoxicaciones Agudas por Aceteminofén

\begin{tabular}{|c|c|}
\hline Etapa & Caracterísiticas \\
\hline Primeras 24 horas & $\begin{array}{l}\text { Síntomas inespecíficos: malestar } \\
\text { general, palidez, náuseas, vómito, } \\
\text { diaforesis. Pruebas de función hepáti- } \\
\text { ca dentro de límites normales }\end{array}$ \\
\hline $\begin{array}{l}\text { II. Entre } 24 \text { y } 48 \text { horas } \\
\text { después de la ingestión }\end{array}$ & $\begin{array}{l}\text { Mejoría sintomática; sin embargo, las } \\
\text { pruebas de función hepática comien- } \\
\text { zan a alterarse }\end{array}$ \\
\hline $\begin{array}{l}\text { III. } 72 \text { a } 96 \text { horas des- } \\
\text { pués de la ingestión }\end{array}$ & $\begin{array}{l}\text { Reaparecen síntomas con mayor } \\
\text { severidad, ocasionalmente acom- } \\
\text { pañados de ictericia o alteraciones } \\
\text { en el estado de conciencia. Puede } \\
\text { evidenciarse compromiso renal o } \\
\text { pancreático y el paciente puede evo- } \\
\text { lucionar a falla hepática fulminante. } \\
\text { Elevación importante de pruebas de } \\
\text { función hepática }\end{array}$ \\
\hline $\begin{array}{l}\text { IV. Después de } 96 \\
\text { horas }\end{array}$ & $\begin{array}{l}\text { Mejoría sintomática con completa } \\
\text { recuperación clínica y de la función } \\
\text { hepática si el paciente recibe un } \\
\text { adecuado manejo }\end{array}$ \\
\hline
\end{tabular}

En los reportes de casos de pacientes con nefropatía inducida por acetaminofén se ha mostrado que se puede presentar falla renal entre el primero y el octavo día, con un pico máximo entre el segundo y el quinto día post ingestión. Aproximadamente el $1 \%$ de estos pacientes llegan a requerir diálisis como mediada temporal, en especial aquellos con falla orgánica multisistémica y otras comorbilidades médicas. La mayoría de pacientes recuperan su función renal totalmente un mes después (14).

No se conoce con exactitud la relación entre la dosis ingerida y el desarrollo de hepatotoxicidad o nefrotoxicidad. La nefrotoxicidad inducida por acetaminofén puede presentarse con dosis tan bajas como las reportadas para hepatotoxicidad (14). Estudios prospectivos recientes han mostrado evidencia de lesión hepática leve con dosis terapéuticas de 1 gramo de acetaminofén cada seis horas en voluntarios sanos (19). Algunos autores sugieren entonces que la hepatotoxicidad por acetaminofén sea considerada con la ingesta de dosis mayores a 4 gramos/día. Se han reportado incluso casos de hepatotoxicidad severa con dosis terapéuticas en pacientes con factores de riesgo (20).

\section{Laboratorio}

La toxicidad del acetaminofén se manifiesta por la presencia de aminotransferasas séricas elevadas (hasta 
de 400 veces por encima de su valor normal), con hipoprotombinemia concomitante, acidosis metabólica y falla renal. La mayor parte de los pacientes presentan niveles séricos normales o levemente elevados de bilirrubinas debido a la lesión hepática (18). En pacientes con intoxicación de origen desconocido se debe realizar un screening para la búsqueda de tóxicos o para sustancias ilícitas en orina y suero.

Debido a la poca especificidad de los síntomas en las primeras horas posteriores a la ingesta del medicamento, se ha evaluado en varios estudios, el papel que tiene la medición de los niveles séricos de acetaminofén en todos los casos de sobredosis intencionales (16-17). Teniendo en cuenta el frecuente uso del acetaminofén, el bajo costo que tienen las pruebas que miden los niveles séricos de este medicamento y las potenciales consecuencias de una sobredosis no reconocida inicialmente, algunos autores recomiendan que a todos los pacientes con sobredosis agudas intencionales o accidentales, se debe incluir en el screening para la búsqueda de tóxicos, el de acetaminofén $(15,21)$.

En los pacientes en los que se tiene la certeza de ser una sobredosis por acetaminofén, la medición de los niveles séricos del medicamento dentro de las primeras 24 horas, permite ver el grado de severidad de la intoxicación; para determinar la cantidad de N-acetilcisteína a utilizar, se usa como parámetro el nomograma de Rumack - Matthews. Es importante anotar que en pacientes con hiperbilirrubinemia (generalmente con bilirrubinas mayores a $10-15 \mathrm{mg} / \mathrm{dl}$ ) se pueden obtener resultados falsos positivos en las pruebas que miden los niveles séricos de acetaminofén (18).

\section{Tratamiento}

El tratamiento debe enfocarse primero a estabilizar al paciente, luego iniciar manejo de soporte, disminuir la absorción del medicamento y detectar el compromiso hepático, para finalmente iniciar el tratamiento específico con el antídoto y manejar las complicaciones.

\section{Manejo general inicial}

- Como cualquier intoxicación, se deben establecer todas las medidas generales con el algoritmo del ABCDE.

- Posteriormente se deben tomar las muestras necesarias para valorar función hepática, función renal, marcadores de coagulación, electrolitos, glucemia etc, y determinar la presencia y niveles séricos del tóxico. En el caso del acetaminofén, los niveles se miden idealmente cuatro horas después de la ingesta (tiempo de distribución); este valor se correlaciona directamente con la severidad de la intoxicación, el riesgo de hepatotoxicidad y la indicación de manejo con $\mathrm{N}$-acetil cisteína.

- Durante las primeras cuatro horas posteriores a la ingesta se recomienda realizar evacuación del contenido gástrico para disminuir la absorción del acetaminofén, con la utilización de lavado gástrico, carbón activado o catárticos. El uso de carbón activado es discutido por algunos autores ya que podría interferir con la absorción de la $\mathrm{N}$-acetilcisteína. Es importante recordar durante la anamnesis, determinar la existencia de factores de riesgo que pueden influir en una mayor hepatotoxicidad.

\section{Manejo específico}

El antídoto para la intoxicación por acetaminofén es la $\mathrm{N}$-acetilcisteína (precursor del glutatión), la cual tiene dos mecanismos de acción: en pacientes con ingestiones agudas menores de ocho horas, tiene un mecanismo preventivo al restituir los niveles hepáticos de glutatión, lo cual lleva a la detoxificación de los metabolitos tóxicos o a impedir su formación(15); por otro lado, en pacientes que ya presentan evidencia clínica y paraclínica de hepatotoxicidad inducida por acetaminofén, probablemente contribuye a repaprar el daño oxidativo al reponer los depósitos de glutatión y generar cisteína (12); sin embargo, este efecto es menos conocido.

La N-acetilcisteína administrada en las primeras ocho horas después de la ingestión de una dosis potencialmente tóxica de acetaminofén, es extremadamente efectiva, aunque su administración se puede realizar incluso hasta las primeras 36 horas post ingestión o si el paciente evoluciona hacia un incremento en el riesgo de toxicidad hepática determinado por el monitoreo de los niveles de acetaminofén $(27,28,29)$. Hay evidencia de que la N-acetilcistína es útil para iniciar el manejo, sin importar el tiempo transcurrido ni la profundidad aparente de los efectos tóxicos. En etapas tardías se ha propuesto como mecanismo de acción, mejorar la liberación de oxígeno por medio de un aumento en la circulación hepato esplénica y un aumento en la extracción de oxígeno (30-32). 


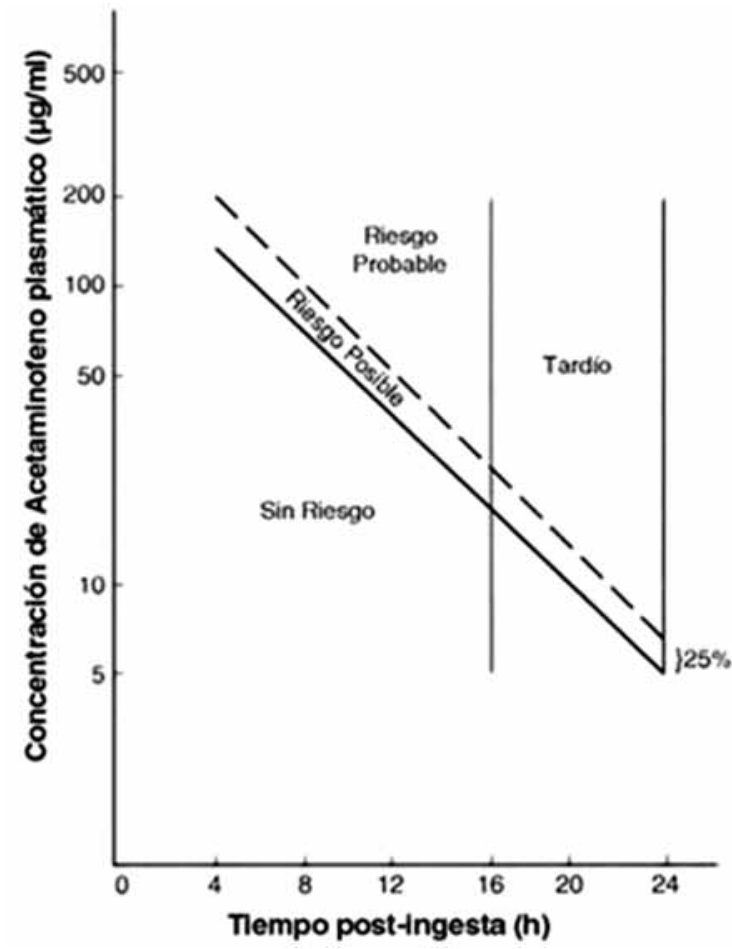

Figura 1. Nomograma Modificado de Rumack-Matthew. Modificado de: Zed, Peter J.

Treatment of acetaminophen overdose. Am J Health-Syst Pharm. 1999; 56:1081-93

El nomograma de Rumack Mathew es una herramienta que se usa como guía de manejo después de una sobredosis aguda de acetaminofén. Según los primeros estudios realizados con este nomograma, los niveles séricos de acetaminofén que están por encima de la línea trazada para conectar el punto correspondiente a la concentración de $200 \mathrm{mg} / \mathrm{ml}$, obtenida a las cuatro horas post ingestión, al correspondiente a $6,25 \mathrm{mg} / \mathrm{ml}$ obtenida a las 24 horas post ingestión, se asociaban o eran predictivos de hepatotoxicidad. Esta línea se conoce como línea de probable toxicidad (24). En Europa, estos valores se utilizan como indicación para tratamiento con $\mathrm{N}$-acetilcisteína (15). En Estados Unidos, la FDA disminuyó el umbral de tratamiento en este nomograma en un $25 \%$, y se considera como línea de posible toxicidad aquella trazada para conectar el punto correspondiente a la concentración de $150 \mathrm{mg} /$ $\mathrm{ml}$ de acetaminofén obtenida a las cuatro horas post ingestión, al correspondiente a $4,7 \mathrm{mg} / \mathrm{ml}$ obtenida a las 24 horas post ingestión.

El periodo de tiempo inicial de cuatro horas post ingestión fue escogido arbitrariamente, esperando un tiempo prudencial para la completa absorción y pico sérico del medicamento. En las ingestiones agudas con tiempo de evolución mayor a 24 horas, la línea en el nomograma se acerca al límite inferior de detección de muchos laboratorios; en estos casos, la decisión de iniciar manejo con $\mathrm{N}$-acetilcisteína se tomará teniendo en cuenta los niveles de transaminasas, niveles séricos del medicamento, la cantidad ingerida por el paciente, y los factores de riesgo para toxicidad.

Generalmente los pacientes con factores de riesgo que toman dosis supraterapéuticas pueden presentar hepatotoxicidad. Si estos pacientes presentan niveles detectables de acetaminofén y aumento en las transaminasas, o si tienen factores de riesgo importantes a pesar de que resultados de las pruebas del laboratorio sean normales, se recomienda realizar tratamiento con N-acetilcisteína por vía oral o parenteral $(15,25,26)$; el tiempo de administración varía entre 20 y 72 horas, tiempo recomendado en los diferentes protocolos publicados.

El tratamiento estándar con $\mathrm{N}$-acetilcisteína por vía intravenosa por un período de 20 horas se usa desde hace más de 20 años en Europa, Canadá y Australia, y desde el 2004 en Estados Unidos; el protocolo a seguir es: dosis inicial de $150 \mathrm{mg} / \mathrm{Kg}$ en $200 \mathrm{ml}$ de dextrosa $5 \%$ en 15 minutos, seguido de $50 \mathrm{mg} / \mathrm{kg}$ en $500 \mathrm{ml}$ de dextrosa $5 \%$ para pasar en cuatro horas, y terminar con goteo de $100 \mathrm{mg} / \mathrm{kg}$ en $1000 \mathrm{ml}$ de dextrosa $5 \%$ por 16 horas. Diferentes estudios y la larga experiencia obtenida en varios años han mostrado que este protocolo es seguro y efectivo (15), y es el que más se emplea actualmente en nuestro medio; se realiza con $\mathrm{N}$-acetilcisteína oral por un periodo de 72 horas siguiendo el siguiente protocolo: dosis inicial de $140 \mathrm{mg} / \mathrm{kg}$, seguida de 17 dosis de $70 \mathrm{mg} / \mathrm{kg}$ cada cuatro horas.

Este protocolo igualmente ha mostrado ser seguro y efectivo. Los estudios que han comparado los protocolos que recomiendan un tratamiento por 20,48 y 72 horas, no han mostrado ninguna diferencia ni ventaja, cuando se inician en las primeras ocho horas post ingestión del acetaminofén. Aunque existe concenso en que se prefiera la adminstración intravenosa en la fase de vómito intratable de la intoxicación, ningún estudio ha mostrado ventaja de la administración parenteral sobre la vía oral $(33,34)$.

Los efectos adversos reportados para la $\mathrm{N}$-acetilcisteína endovenosa son hipotensión, urticaria y reacciones 
anafilactoides leves y transitorias, las cuales parecen estar relacionadas con la dosis y rata de administración. La frecuencia de presentación de estas reacciones adversas varía en la mayor parte de estudios entre el $3 \%$ al $25 \%(35,36,44)$. Por vía oral los efectos se presentan a nivel gastrointestinal como náuseas y vómito.

Otras medidas a tener en cuenta:

- En caso que el paciente presente signos o síntomas de insuficiencia hepática aguda, encefalopatía grado III-IV, prolongación del tiempo de protombina, disminución de factores $\mathrm{V}$ y VIII, hipertensión endocraneana o acidosis láctica, se debe trasladar a la unidad de cuidado intensivo.

- Establecer indicaciones y posibilidad de transplante hepático en pacientes que desarrollen falla hepática aguda. Las indicaciones para trasplante hepático en casos de intoxicación por este fármaco son:

- $\mathrm{pH}$ arterial menor a 7,3, independientemente del grado de encefalopatía ó

- Encefalopatía grado III o IV y tiempo de protrombina mayor a 100 segundos y creatinina mayor a $3,4 \mathrm{mg} / \mathrm{ldl}$

- En pacientes con falla renal aguda es importante evaluar si es necesario la diálisis.

- Determinar tempranamente la causa de la sobredosis; si fue voluntaria (con intención suicida), es fundamental buscar apoyo en el grupo de psiquiatría.

\section{Pronóstico}

Algunos estudios han mostrado que en los casos de hepatotoxicidad por acetaminofén, los resultados de los análisis clínicos y de laboratorio pueden predecir el pronóstico en estos pacientes (38); si se tiene un INR $>6,5$ (o tiempo de protombina $>1,8$ veces del control), creatinina sérica mayor a $3,3 \mathrm{mg} / \mathrm{dl}$, acidosis metabólica importante $(\mathrm{pH}<7,3$ después de una adecuada reanimación con líquidos) ó encefalopatía grado III o más (King's College Criteria KCC), el pronóstico es pobre $(15,22)$.

El método que se ha empleado como indicador temprano de falla hepática inminente y de necesidad de transplante hepático es el APACHE II. Algunos investigadores han mostrado que esta herramienta es más sensible y específica que los KCC (39); sin embargo, es más densa y difícil de recordar. Se han propuesto otros análisis que podrían emplearse como factores pronósticos en estos pacientes tales como las pruebas que miden los niveles séricos de fosfato (40), los niveles de lactato (41), la hiperamilasemia (42) y los niveles de alfa-fetoproteína (43); sin embargo, se requieren más estudios al respecto.

La morbilidad en la sobredosis de acetaminofén aumenta proporcionalmente con el tiempo que transcurre para la administración de la N-acetilcisteína (45). Si el tratamiento se inicia en las primeras ocho horas, el desarrollo de hepatotoxicidad es extremadamente raro $(<5 \%)$; si se inicia en las primeras 16 a 24 horas, la toxicidad se presenta en aproximadamente el $41 \%$ de los casos $(12,23)$.

\section{Referencias}

1. Larson A. Acetaminophen Hepatotoxicity. Clinics in Liver Disease. 2007;11:525-548.

2. Litovitz T L, Klein-Schwartz W, White S. 1999 Annual Report of the American Association of Poison Control Centers Toxic Exposure Surveillance System. Am J Emerg Med. 2000;18:517-74.

3. Bialas M C, Reid P G, Beck P. Changing Patterns of Selfpoisoning in a UK Health District. QJM. 1996;89:893-901.

4. Watson W A, Litovitz T L, Klein-Schwartz W, Rodgers G C Jr, Youniss J, Reid N, et al. 2003 Annual Report of the American Association of Poison Control Centers Toxic Exposure Surveillance System. Am J Emerg Med. 2004;22:335-404.

5. 2005 Anual Report of the American Association of Poison Control Centers. Availabe from: http//www. aapc.org

6. Ostapowicz G, Fontana R J, Schiodt F V. Results of a Prospective Study of Acute Liver Failure at 17 Tertiary Care Centers in the United States. Ann Intern Med. 2002;137(12):947-54

7. Larson A M, Polson J, Fontana R J. Acetaminophen-induced Acute Liver Failure: Results of a United States Multicenter, Prospective Study. Hepatology. 2005;42(6):1364-72.

8. Brunton L, Parker K, Blumenthal D, Buxton I. Goodman \& Gilman's Manual of Pharmacology and Therapeutics. 2008.

9. Khandar M A, Parmar D V, Das M, Katyare S S. Is Activation of Lysosomal Enzymes Responsible for Paracetamol-induced Hepatotoxicity and Nephrotoxicity? J Pharm Pharmacol. 1996:48:437-40.

10. Lorz C, Justo P, Sans AB, Egidio J, Ortiz A. Role of Bcl-xl in Paracetamol-induced Tubular Epithelial Cell Death. Kidney Int. 2005;67:592-601.

11. William M L. Drug Induced Hepatotoxicity. N Engl J Med. 2003;349:474-85.

12. Sisamon I. Acerca de la Hepatotoxicidad del Paracetamol. Revista del Hospital Privado de Comunidad. 2003;6(2).

13. Prescott L F. Paracetamol Overdosage: Pharmacological Considerations and Clinical Management. Drugs. 1983;25:290-314.

14. Mazer M, Perrone J. Acetaminophen-Induced Nephrotoxocity: Pathopysiology, Clinical Manifestation and Management. $J$ Medical Toxicology. 2008;4(1).

15. Rowden A, Norvell J, Eldridge D, Kirk M. Acetaminophen Poisoning. Clinics in Laboratory Medicine. 2006;26:49-65.

16. Ashbourne J F, Olson K R, Khayam-Bashi H. Value of Rapid Screening for Acetaminophen in All Patients with Intentional Drug Overdose. Ann Emerg Med. 1989;18(10):1035-8. 
17. Sporer K, Khayam-Bashi H. Acetaminophen and Salicylate Serum Levels in Patients with Suicidal Ingestion or Altered Mental Status. Am J Emerg Med. 1996;14(5):443-6.

18. Fontana R. Acute Liver Failure Including Acetaminophen Overdose. Med Clin N Am. 2008;92:761-94.

19. Watkins P B, Kaplowitz N, Slattery J. Aminotranferase Elevation in Healty Adults Receiving 4 Grams of Acetaminophen Daily. JAMA. 2006;296:87-93.

20. Moling O, Cairon E, Rimenti G, Rizza F, Pristera R, Mian P. Severe Hepatotoxicity after Therapeutic Doses of Acetaminophen. Clinical Therapeutics. 2006;28(5):755-60.

21. Eldridge D, Holstege C. Utilizing the Laboratory in the Poisoned Patient. Clin Lab Med. 2006;26:13-30.

22. O'Grady J, Alexander G. Early Indicators of Prognosis in Fulminant Hepatic Failure. Gastroenterology. 1989;97:439.

23. Rumack B H. Acetaminophen Overdose, 662 Cases with Evaluation of NAC. Arch Intern Med. 1981;5:871.

24. Rumack B H, Mathew H. Acetaminophen Poisoning and Toxicity. Pediatrics. 1975;55(6):871-6.

25. Kozer E, Barr J, Bulkowstein M. A Prospective Study of Multiple Supratherapeutic Acetaminophen Doses in Febrile Children. Vet Humn Toxicol. 2002;44(2):106-9.

26. Daly F F, O'Malley G F, Heard K. Prospective Evaluation of Repeated Supratherapeutic Acetaminophen Ingestion. Ann Emerg Med. 2004;44(4):393-8.

27. Smilkstein M J, Knapp G L, Kulig K W. Efficacy of Oral NAcetylcysteine in the Treatment of Acetaminophen Overdose. Analysis of the National Multicenter Study (1976 to 1985). N Engl J of Med. 1988;319(24):1557-62.

28. Prescott L F, Illingworth R N, Critchley J A. Intravenous N-acetylcysteine: The Treatment of Choise for Paracetamol Poisoning. BMJ. 1979;2(6198):1097-100

29. Peterson R G, Rumack B H. N-Acetylcysteine for Acetaminophen Overdosage. N Eng J Med. 1977;296(9):515.

30. Keays R, Harrison P M, Wendon J A. Intravenous N-Acetylcysteine in Paracetamol Induced Fulminant Hepatic Failure: A Prospective Controlled Trial. BMJ. 1991;303(6809):1026-9.

31. Spies C D, Reinhart K, Witt I. Influence of N-Acetylcysteine on Indirect Indicators of Tissue Oxigenation in Septic Shock Patients: Results from a Prospective, Randomized, Double Bind Study. Crit Care Med. 1994;22(11):1738-46.
32. Devlin J, Ellis AE, McPeake J. N-Acetylcysteine Improves Indocyanine Green Extraction and Oxygen Transport During Hepatic Dysfunction. Crit Care Med. 1997;25(2):236-42.

33. Taylor S E. Acetaminophen Intoxication and Length of Treatment: How Long is Long Enough? A Coment. Pharmacotherapy. 2004;24(5):694-6.

34. Buckley N A, Whyte I M, Conell D L. Oral or Intravenous NAcetylcysteine: Which is the Treatment of Choice for Acetaminophen Poisoning? J Toxicol Clin Toxicol. 1999;37(6):759-67.

35. Lynch R M, Robertson R. Anaphylactoid Reactions to Intravenous N-Acetylcysteine: A Prospective Case Controlled Study. Accid Emerg Nurs. 2004;12(1):10-5.

36. Mullins M E, Schmidr R U Jr, Jang T B. What is the Rate of Adverse Events with Intravenous Versus Oral N-Acetylcysteine in Pediatric Patients? Ann Emerg Med. 2004;44(5):547-9.

37. Peter J. Treatment of Acetaminophen Overdose. Am J HealthSyst Pharm. 1999;56:1081-93.

38. O'Grady J G, Alexander G J, Hayllar K M. Early Indicators of Prognosis in Fulminant Hepatic Failure. Gastroenterology. 1989;97(2):439-45.

39. Mitchell I, Bihari D, Chan R. Earlier Identification of Patients at Risk from Acetaminophen Induced Acute Liver Failure. Crit Care Med. 1998;26(2):279-84.

40. Schmidt LE, Dalhoff K. Serum Phosphate is an Early Predictor of Outcome in Severe Acetaminophen-induced Hepatotoxicity. Hepatology. 2002;36(3):659-65.

41. Bernal W, Donaldson N, Wincoll D. Blood Lactate as an Early Predictor of Outcome in Paracetamol-induced Acute Liver Failure: A Cohort Study. Lancet. 2002;359(9306):558-63.

42. Schmidt L E, Dalhoff K. Hyperamylasemia and Acute Pancreatitis in Paracetamol Poisoning. Aliment Pharmacol Ther. 2004;20(2):173-9.

43. Schmidt L E, Dalhoff K. Alpha-fetoprotein is a Predictor of Outcome in Paracetamol Induced Liver Injury. Hepatology. 2004;41(1):26-31.

44. Whyte A, Kehrl T, Brooks D, Katz K, Sokolowski D. Safety and Effectiveness of Acetadote for Acetaminophen Toxicity. $J$ Emerg Med. 2008

45. Schilling A, Corey R, Leonard M, Eghtesad B. Acetaminophen: old Drug, New Warnings. Cleve Clin J Med. 2010;77:19-27. 